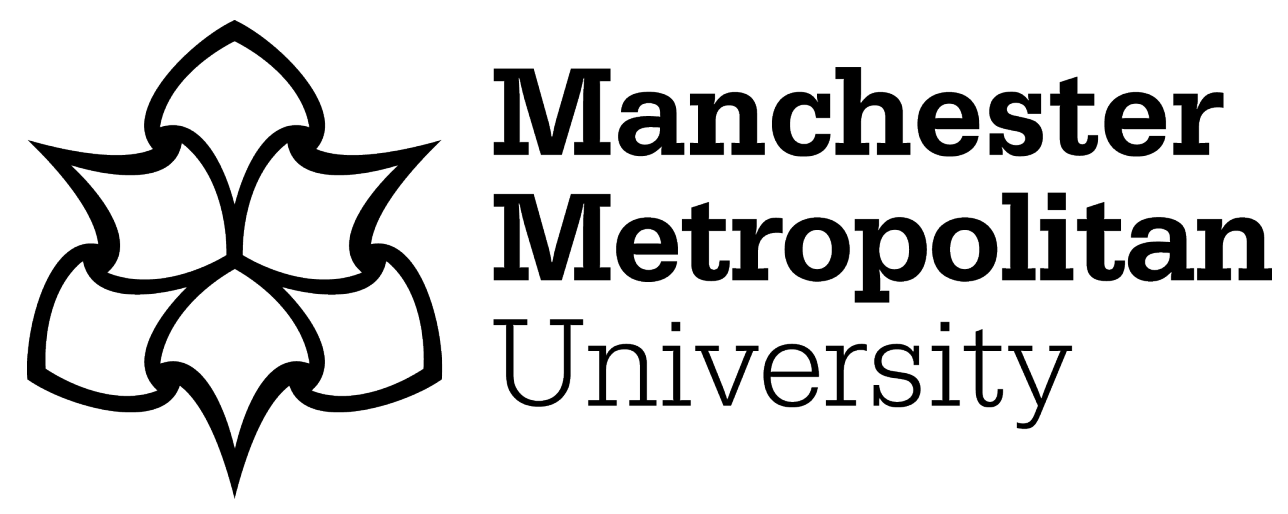

Cumaraswamy, AA, Lewis, AM, Geletu, M, Todic, A, Diaz, DB, Cheng, XR, Brown, CE, Laister, RC, Muench, D, Kerman, K, Grimes, HL, Minden, MD and Gunning, PT (2014) Nanomolar-potency small molecule inhibitor of STAT5 protein. ACS Medicinal Chemistry Letters, 5 (11). pp. 1202-1206. ISSN 1948-5875

Downloaded from: https://e-space.mmu.ac.uk/624261/

Version: Accepted Version

Publisher: American Chemical Society

DOI: https://doi.org/10.1021/ml500165r

Please cite the published version 


\title{
Nanomolar-Potency Small Molecule Inhibitor of STAT5 Protein
}

\author{
Abbarna A. Cumaraswamy, ${ }^{\dagger, \perp}$ Andrew M. Lewis, ${ }^{\dagger, \perp}$ Mulu Geletu, ${ }^{\dagger, \perp}$ Aleksandra Todic, ${ }^{\dagger}$ Diego B. Diaz, \\ Xin Ran Cheng, ${ }^{\ddagger}$ Carla E. Brown, ${ }^{\dagger}$ Rob C. Laister, ${ }^{\|}$David Muench, ${ }^{\S}$ Kagan Kerman, ${ }^{\ddagger}$ \\ H. Leighton Grimes, ${ }^{\S}$ Mark D. Minden, $"$ and Patrick T. Gunning ${ }^{*}{ }^{\dagger}$ \\ ${ }^{\dagger}$ Department of Chemistry, University of Toronto Mississauga, Mississauga, Ontario L5L 1C6, Canada \\ ${ }^{\ddagger}$ Department of Physical \& Environmental Sciences, University of Toronto at Scarborough, Toronto, Ontario M1C 1A4, Canada \\ ${ }^{\S}$ Division of Immunobiology, Cincinnati Children's Hospital Medical Center, Cincinnati, Ohio 45229, United States \\ "Princess Margaret Cancer Centre, Ontario Cancer Institute, 610 University Avenue, Toronto, Ontario M5G 2M9, Canada
}

Supporting Information

\begin{abstract}
We herein report the design and synthesis of the first nanomolar binding inhibitor of STAT5 protein. Lead compound 13a, possessing a phosphotyrosyl-mimicking salicylic acid group, potently and selectively binds to STAT5 over STAT3, inhibits STAT5-SH2 domain complexation events in vitro, silences activated STAT5 in leukemic cells, as well as STAT5's downstream transcriptional targets, including $M Y C$ and MCL1, and, as a result, leads to apoptosis. We believe 13a represents a useful probe for interrogating STAT5 function in cells as well as being a potential candidate for advanced preclinical trials.
\end{abstract}

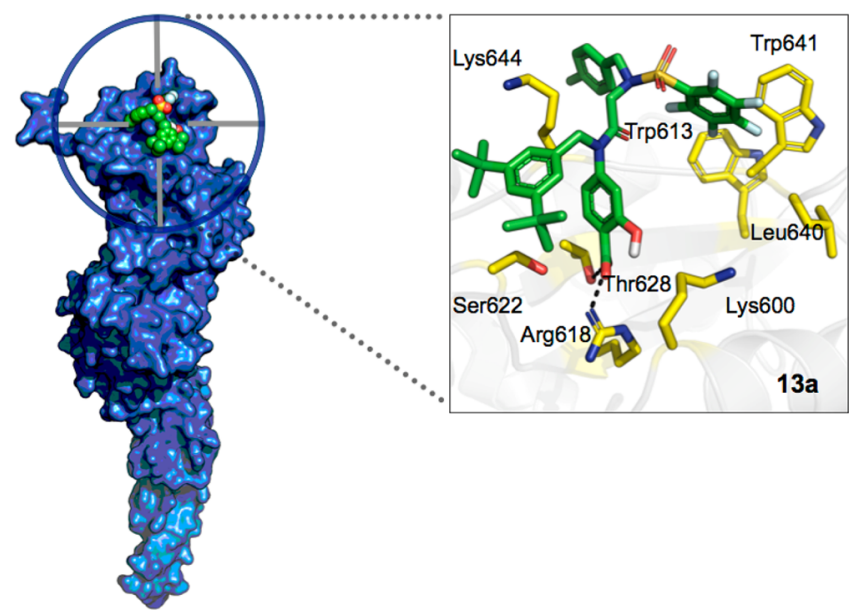

KEYWORDS: STAT5, small-molecule inhibitor, protein-protein interactions, anticancer drug, leukemia cells

$\mathrm{N}$ umerous inhibitors have been developed to target the JAK-STAT signaling pathway, a major driving force in hematopoietic malignancies. While emphasis has focused on identifying effective upstream kinase inhibitors for suppressing STAT activity, inhibitors have suffered from poor kinase selectivity, ${ }^{1}$ cardiovascular toxicity, ${ }^{2,3}$ and, in some clinical cases, acquired resistance. ${ }^{4}$ Multiple kinase inhibitors have been used in combination to try to combat resistance. ${ }^{5,6}$ An alternative strategy to reduce off-target toxicity is to target proteins immediately downstream of the kinases, such as the Signal Transducer and Activator of Transcription (STAT) 5 protein. STAT5 is thus a compelling molecular target for therapeutic intervention.

In normal cells, the activation of STAT5 proteins is tightly regulated by cytokines (IL-2, IL-5, IL-7, GM-CSF, erythropoietin (EPO), thrombopoietin, and prolactin) and growth factors. ${ }^{7}$ Binding of these extracellular ligands to their target receptors induces the activation of receptor-associated JAK kinases that phosphorylate key tyrosine residues within the receptor, providing docking sites for the SRC homology 2 (SH2) domains of the inactive cytoplasmic STAT5 monomers. STAT5 is then phosphorylated at specific tyrosine residues, either Y694 (STAT5A) or Y699 (STAT5B) of the C-terminus. Phosphorylated STAT5 monomers form either homo- or hetero- ${ }^{8,9}$ STAT5X-STATX dimers through reciprocal phosphotyrosine-SH2 interactions. Activated STAT5 dimers translocate to the nucleus, where they bind to STAT5 DNA response elements inducing transcription of genes involved in proliferation ( $\mathrm{Bcl}-\mathrm{xl}, \mathrm{c}-\mathrm{Myc}$, pim-1), cell differentiation ( $\mathrm{p} 21)$, cell survival (MCL-1) inflammation (Osm), and apoptosis (JAB). ${ }^{10}$ In contrast, mutations within cytosolic kinases (TELJAK2, Bcr-Abl, FLT-3) as well as overactive receptor-associated tyrosine kinases (SRC, EGFR) induce constitutive phosphorylation of STAT5 proteins, increasing the production of antiapoptotic genes, which can contribute to driving the cancer phenotype. $^{11}$

Approaches aimed at directly targeting STAT5 have been limited to a chromone-derived acyl hydrazine inhibitor, identified through a high-throughput fluorescence polarization (FP) screen. While this agent exhibited promising in vitro disruption of the STAT5:EPOR phosphopeptide interaction, higher concentrations were required to inhibit STAT5 in cells. ${ }^{12}$ A STAT5 function-based screening approach identified

Received: April 27, 2014

Accepted: September 19, 2014

Published: September 19, 2014 
the FDA approved neuroleptic drug, pimozide, as a potent inhibitor of STAT5 phosphorylation, resulting in the downregulation of STAT5 target gene expression in $\mathrm{Ba} / \mathrm{f} 3$ FLT3 ITD cells. The authors believe pimozide is an effector of negative regulators that modulate STAT5 phosphorylation. ${ }^{13,14}$

We have previously prepared a library of phosphopeptidemimicking salicylic acid-based compounds to target STAT3's $\mathrm{SH} 2$ domain. Screening against STAT5 via $\mathrm{FP}^{15}$ identified compounds 1 and 2 as STAT5 binders with 2-3-fold selectivity over STAT3 (Figure 1). In whole cells, 1 showed selective

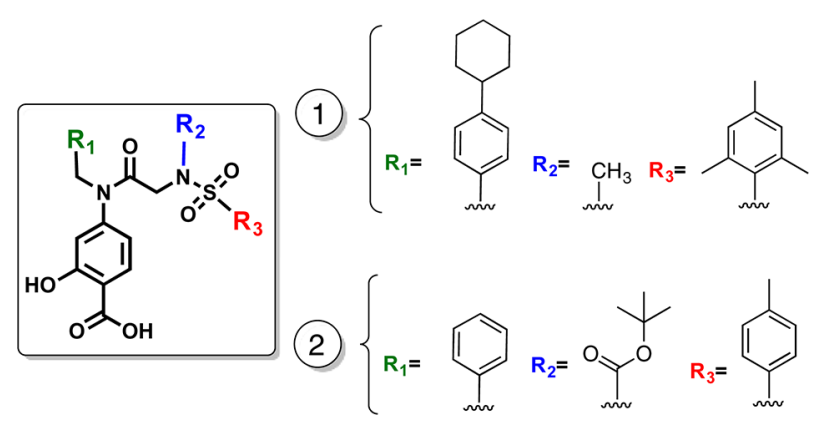

Figure 1. STAT5 inhibitors BP-1-108 (1) and SF-1-088 (2).

suppression of STAT5 Tyr phosphorylation $\left(\mathrm{IC}_{50} \sim 20 \mu \mathrm{M}\right.$ ) and STAT5 target genes, MYC and CCND1, at $\mu \mathrm{M}$ concentrations $(\sim 40 \mu \mathrm{M}))^{16}$ However, progress toward a potent and selective nanomolar (nM) inhibitor of STAT5 has been slow. Herein, we describe the identification of the first $\mathrm{nM}$, STAT5-selective inhibitor using in silico-directed efforts. ${ }^{17}$

Scaffolds 1 and $\mathbf{2}$ were selected as a starting point for structural optimization. Interestingly, both leads possessed opposing lipophilic trends for the substituents found at the $R_{1}$ position. Increasing lipophilicity and bulk at $\mathrm{R}_{1}$ seems to favor selectivity for the STAT3 protein. However, bulky hydrophobic substituents on the $R_{2}$ position seem to dictate the largest selectivity for the STAT5 protein, suggesting structural variability within the STAT3 and STAT5 SH2 domains. ${ }^{16}$ To exploit these observed differences, we probed the $\mathrm{SH} 2$ domain pocket using computational docking simulations.

While the activated STAT5-STAT5 dimer structure has yet to be solved, the pY binding pocket of the $\mathrm{SH} 2$ domain was identified by comparing and contrasting the structural architectures and binding sites of $121 \mathrm{SH} 2$ domain-containing proteins. ${ }^{18} \mathrm{SH} 2$ domains are defined by an antiparallel $\beta$-sheet flanked by two $\alpha$ helices. The pY binds in a pocket located within the $\beta \mathrm{B}, \mathrm{C}$, and $\mathrm{D}$ strands. Specifically, a conserved $\mathrm{R}(\mathrm{XXX})$ residue on the $\beta \mathrm{B}$ strand participates in electrostatic interactions with pY. ${ }^{19}$ Notably, STAT3's structure was resolved in both the unphosphorylated (PDB: 3CWB) and phosphorylated (PDB: 1BG1) states. $^{20,21}$ Superimposition of structures revealed that STAT3's SH2 domain structure is not significantly altered upon phosphopeptide binding. ${ }^{22}$ Thus, we reasoned that the unphosphorylated STAT5 structure (PDB: $1 \mathrm{Y} 1 \mathrm{U}$ ) is suitable for in silico-based drug design. Analogous to the canonical pY-SH2 domain binding, the STAT5 pY likely docks proximal to the conserved R618 ( $\beta$ B strand), making $\mathrm{H}$ bonding/electrostatic interactions with nearby polar residues, $\mathrm{K} 600(\alpha \mathrm{A}), \mathrm{T} 628(\beta \mathrm{C})$, and $\mathrm{S} 622(\beta \mathrm{B}$ and $\beta \mathrm{C})$ Figure $2 \mathrm{~A}$.

Next, using AutoDock4.2, we performed global searches of the conformational space along with careful local searches to derive the best conformational fit within the STAT5 SH2 domain. Docking simulations were carried out with a rigid
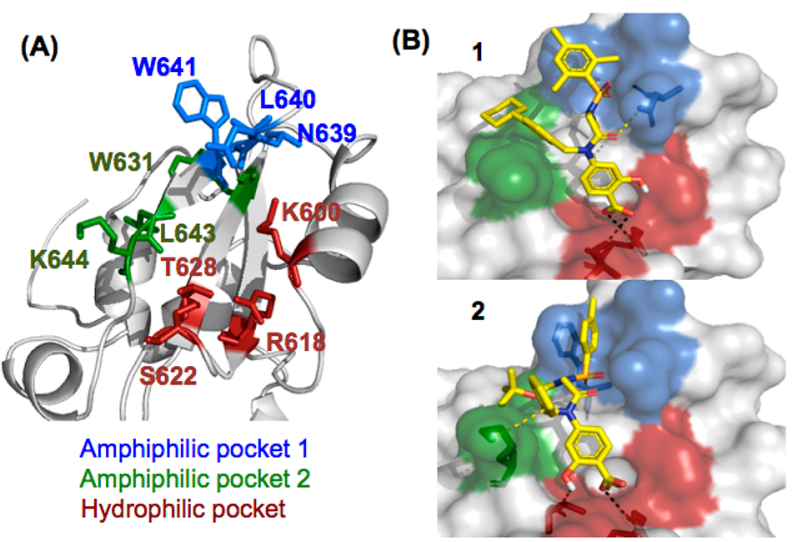

Figure 2. (A) STAT5a's $\mathrm{SH} 2$ domain with three binding pockets: hydrophilic, red; amphiphilic, green; amphiphilic, blue; (B) in silico docking of 1 interacting with R618, S622, and N639; 2 interacting with R618 and S622, as well as a cationic $-\pi$ interaction of the $R_{1}$ benzyl with K644.

protein structure, allowing for ligand flexibility using a Lamarckian Genetic Algorithm (GA) with the global and adaptive local search parameters through 50 trials of the "long" GA runs. 1 and 2 occupied two amphiphilic pockets. The first pocket contained residues N639, L640, and W641 (blue region, Figure 2A,B), which interact with the sulfonamide mesityl and toluyl of $\mathbf{1}$ and 2, respectively. The adjacent pocket containing W631, L643, and K644 (green) was found to interact with the $\mathrm{R}_{1}$ substituents of both leads. Interestingly, for $2\left(\mathrm{R}_{1}=\mathrm{Ph}\right)$, docking poses showed favorable cationic $-\pi$ interactions with $\mathrm{K} 644$. In contrast, the binding of $\mathbf{1}\left(\mathrm{R}_{1}=\right.$ cyclohexylphenyl) was dominated by van der Waal's interactions with L643. Since the K644 residue is unique to STAT5, we elected to prepare analogs possessing hydrophobic, electron rich, aromatic $R_{1}$ substituents to derive selectivity. The $R_{3}$ substituents were carried forward from previous SAR studies, owing to their favorable biological profiles.

To survey the $\mathrm{R}_{1}$ binding pocket, we explored 24 substituents of varying size and chemical diversity, including furan $(\mathbf{8 a}, \mathbf{m})$, thiophene $(\mathbf{8 b}, \mathbf{n})$, imidazole $(\mathbf{8 c}, \mathbf{o})$, cyclopropyl $(\mathbf{8 d}, \mathbf{p})$, and bicyclo[2.2.1] heptane $(\mathbf{8 e}, \mathbf{q})$ heterocycles, naphthyl, and phenyl; these were chosen for their small size and rich electronic character, instead of the cyclohexylphenyl moiety of 1 (Table 1). The $\mathrm{R}_{3}$ position was left either as a mesityl or pentafluorobenzyl substituent. The tolyl group of $\mathbf{2}$ was not retained due to poor solubility.

The library was screened through a previously reported highthroughput STAT5B FP assay which measures the disruption of phosphopeptide-STAT5B SH2 domain interactions. ${ }^{15} \mathrm{FP}$ measurements were taken every $15 \mathrm{~min}$ for an hour against the fluorescein conjugated phosphopeptide-STAT5B complex to verify that the phosphopeptide probe (5-FAM-GpYLVLDKW) was not being displaced over time.

Time intervals identified the optimal time point at which the maximum change in fluorescence polarization was observed. In general, the smaller heterocyclic $R_{1}$ substituents had no observable activity at inhibitor concentrations up to $100 \mu \mathrm{M}$.

In contrast, only aromatic derivatives $\mathbf{8 j}, \mathbf{8 l}, \mathbf{8 v}$, and $\mathbf{8 x}$ demonstrated similar potencies relative to the parent leads, with $K_{\mathrm{i}}$ values of $6.62,4.77,12.36$, and $11.51 \mu \mathrm{M}$, respectively (Supporting Information Figure S4 and Table S1). We noted that the electron rich, hydrophobic di-tert butyl benzyl groups of $\mathbf{8 1}$ and $8 \mathbf{x}$ consistently engaged in cation $-\pi$ interactions with 
Table 1. Focused Library of 24 Derivatives with Small Heterocycles and Substituted Phenyl Groups at the $\mathbf{R}_{1}$ Position, with Corresponding $K_{i}$ Values Determined through FP

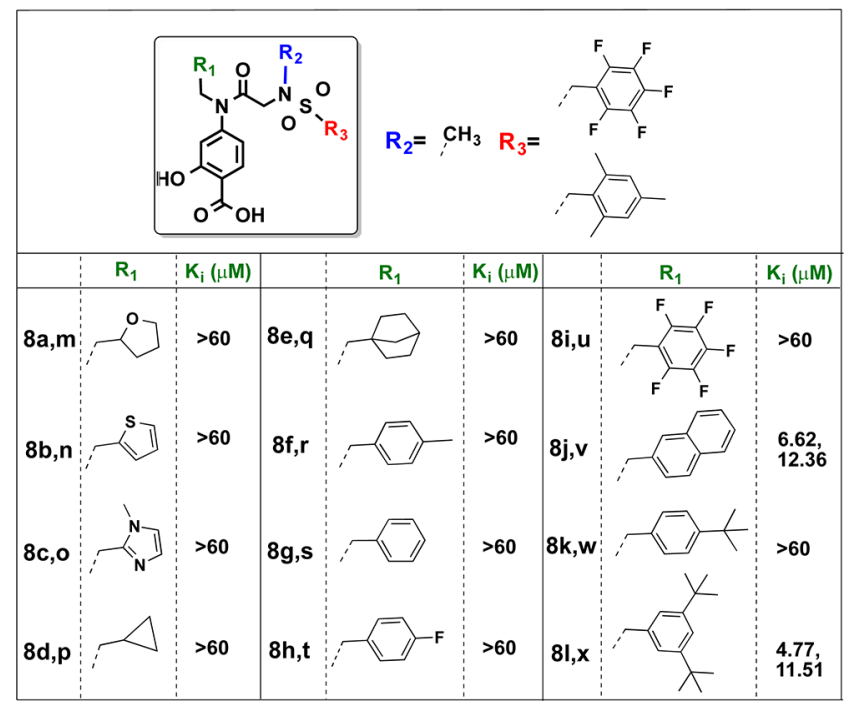

K644, more so than the corresponding heterocyclic derivatives. The naphthyl derivatives, $8 \mathbf{j}$ and $\mathbf{8 v}$, favored $\pi-\pi$ stacking interactions with W641 and not with K644 (Supporting Information Figures S9-12). Introduction of small heterocycles abolished activity. To investigate whether the $N$-boc $\mathrm{R}_{2}$ substituent of 2 might contribute to STAT5 selectivity, we prepared a new series of tetrapodal inhibitors. Di-tert-butyl benzyl and naphthyl substituents at $\mathrm{R}_{1}$ were retained for SAR analysis. In silico experiments showed that the boc group in 2 interacted with residues L643, W631, and W641 (Figure 2B, green region). Since this region contained predominantly electron-rich aromatic residues (W), we hypothesized that agents equipped with electron-deficient $R_{2}$ aromatic groups such as a $p$-chlorobenzyl may favor strong $\pi-\pi$ stacking interactions. Thus, a focused library $13 \mathbf{a}-\mathbf{d}$ was prepared and evaluated via FP against both STAT5 and STAT3 at $1-60,000$ $\mathrm{nM}$ (Supporting Information Figure S5). Most encouragingly, 13a was found to potently disrupt phosphopeptide-STAT5B interactions, $K_{i}=145 \mathrm{nM}$. Moreover, 13a was 1000 -fold more selective for STAT5B cf. STAT3, with a STAT3 $K_{i}=143 \mu \mathrm{M}$ (Figure 3B). In silico docking simulations revealed that 13a, via the $p$-chlorobenzyl and pentafluorobenzene groups, made favorable contacts with the two adjacent amphipathic pockets (Figure 3A, and Figure S13).

In addition to FP, surface plasmon resonance (SPR) binding experiments were conducted to measure the kinetic association and dissociation using a ProteOn XPR36 (Biorad) with fulllength His-tagged, STAT5, and STAT3 (SignalChem) immobilized on a biosensor chip. Most encouragingly, inhibitors exhibited potent nM binding affinities for STAT5, and selectivity for the STAT5 cf. STAT3 (Supporting Information Figure S2). Compound 13a exhibited the most potent $K_{D}\left(k_{o f f} / k_{o n}\right)$ of $42 \pm 4 \mathrm{nM}$, with 7-fold selectivity for STAT5 cf. STAT3, $K_{\mathrm{D}}=287 \pm 29 \mathrm{nM}$ (Figure 3C). As assessed by FP and SPR, 13a represents the first nM STAT5binding, STAT5-selective inhibitor.

To determine the selectivity of 13a for STAT5, we screened for off-target kinase activity, a possible alternative target for an effector of STAT5 phosphorylation. 13a was assessed against
(A)

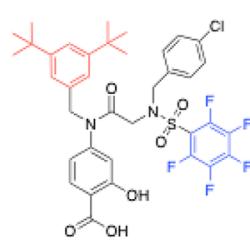

13a

(B)

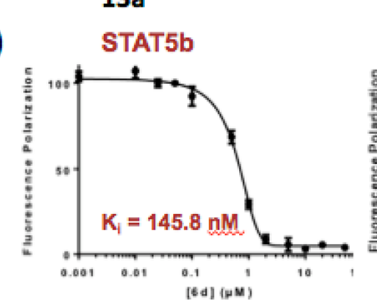

(C)

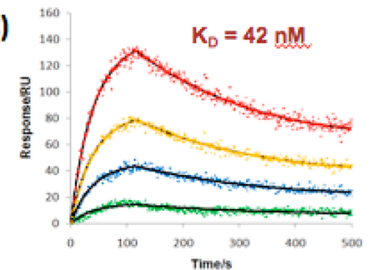

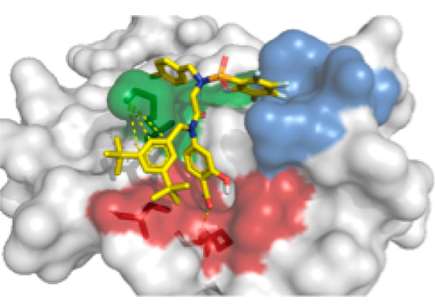

STAT3
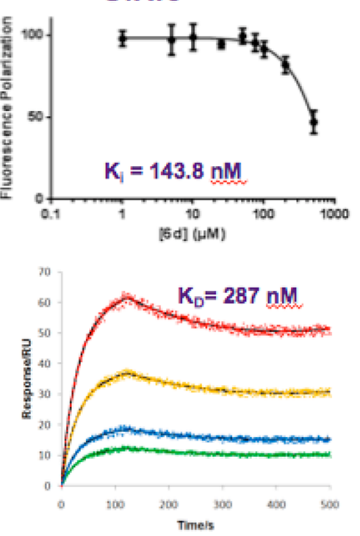

Figure 3. (A) 13a docked with STAT5b; (B) FP binding traces for 13a against STAT5b and STAT3 protein; (C) SPR curves for 13a against STAT5b and STAT3 $(5,1.67,0.56,0.19 \mu \mathrm{M})$.

120 representative kinases covering the diversity of the kinase families (DiscoveRx). Ultrasensitive quantitative PCR (qPCR) was used to measure levels of immobilized kinases after treatment with $13 \mathrm{a}$ at $5 \mu \mathrm{M}$. Encouragingly, 13a showed negligible effects against the bank of kinases and, more notably, against upstream STAT5 activating kinases, JAK1/2, ABL, and FLT-3 (Supporting Information Figure S1). These data suggest that inhibition of pSTAT5 is due to interaction with STAT5's $\mathrm{SH} 2$ domain and not through inhibition of upstream kinases.

Next, 13a was assessed for whole cell potency against STAT5 transformed CML and AML cell lines, K562 (Bcr-Abl) and MV-4;11 (FLT3-ITD), respectively. Cell viability was assessed following treatment at various concentrations of inhibitor $(0.78-50 \mu \mathrm{M})$ using a CellTiter-Blue cell viability assay $(72 \mathrm{~h})$. As compared to first generation STAT5 inhibitors $(\mathbf{8 j}, \mathbf{8 1}, \mathbf{8 v}$, $\mathbf{8 x}), \mathrm{IC}_{50}$ values for $\mathbf{1 3} \mathbf{a}-\mathbf{d}$ were $2-3$-fold higher in potency, with activities ranging from 3 to $20 \mu \mathrm{M}$ (Supporting Information Figures S14, S15). Encouragingly, 13a displayed the most potent activity in FLT3-ITD driven MV4;11 cells, $\mathrm{IC}_{50}=3.5 \mu \mathrm{M}$. We next evaluated 13a-mediated inhibition of STAT5 phosphorylation levels. K652 leukemia cells were treated with 13a for $5 \mathrm{~h}$, the cells were harvested, and the levels of phosphorylated STAT5 (Y694) were determined (Figure 4A). 13a decreased pSTAT5 in a dose dependent manner and ablated pSTAT5 above $10 \mu \mathrm{M}$ with no change in the total STAT5 concentration or cleavable PARP-1. However, K562 cells were found to undergo cell death (cleaved PARP-1, Caspase-3) at $24 \mathrm{~h}$ at $15 \mu \mathrm{M}$, indicating that 13a might induce apoptosis as a result of STAT5 inactivation (Figure 4B). To investigate selectivity, MDA-MB-231 breast cancer cells, which harbor high pSTAT3 and negligible pSTAT5 activity (Supporting Information, Figure S18), were assessed for differential pSTAT inhibition by 13a (Figure 4C). Encouragingly, pSTAT3 was not inhibited at doses corresponding to pSTAT5 inhibition within the leukemic cell line and neither 


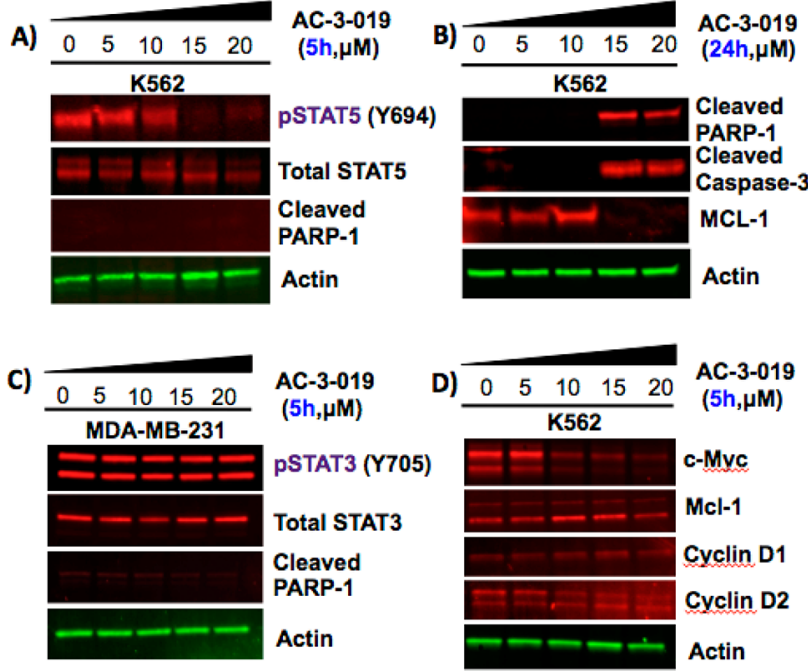

Figure 4. (A) Inhibition of pSTAT5 with 13a $(15 \mu \mathrm{M})$ in K562 cells; (B) Initiation of apoptosis after $24 \mathrm{~h}$ and knockdown of MCL-1 (15 $\mu \mathrm{M})$; (C) 13a shows no effect on pSTAT3 in MDA-MB-231 cells up to $20 \mu \mathrm{M}$; (D) Knockdown of downstream target c-Myc after $5 \mathrm{~h}$.

total STAT3 or STAT5 protein levels were affected. It was shown that 13a was 3-fold less cytotoxic in MDA-MB-231 $\left(\mathrm{IC}_{50}=10 \mu \mathrm{M}\right)$ than in the high pSTAT5 leukemic cell line (Supporting Information Figure S16). Downstream of STAT5, we assessed for modulation of the STAT5 transcriptional targets, MCL-1, CYCLIN D1/D2, and MYC. We reasoned that 13a should decrease gene expression and induce apoptosis by 24 h. K562 cells were dosed with 13a at the same concentrations observed for selective STAT5 inhibition. At 5 $\mathrm{h}$, we observed dose-dependent decreases in MYC and complete knockdown of MCL-1 observed at $24 \mathrm{~h}$ at $15 \mu \mathrm{M}$ (Figure 4D).

We evaluated 13a in healthy human $\mathrm{CD} 34^{+}$umbilical cord cells to determine off-target effects and the therapeutic window. Most encouragingly, there existed an approximate order of magnitude difference in senstivity, with little reduction in cell viability at $10 \mu \mathrm{M} \mathrm{13a}$, while MV4-11 cells were abolished at the same concentrations (Figure 5).

In summary, we have identified the first $\mathrm{nM}$ binding inhibitor of STAT5 protein. Moreover, lead compound 13a has been shown to potently and selectively disrupt STAT5-phosphopeptide interactions $(\mathrm{nM})$ as compared to STAT3 $(\mu \mathrm{M})$. With no off-target kinase activity, 13a was shown to suppress pSTAT5

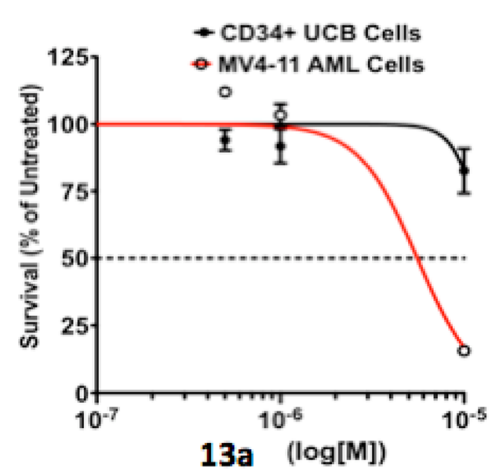

Figure 5. Healthy human CD34+ umbilical cord cells treated with compound 13a at $10 \mu \mathrm{M}$ with limited effect on cell viability in comparison to MV4;11 cells. while having no effect on pSTAT3. Thus, 13a represents a useful probe for interrogating the STAT5 function in diseased cells as well as being a candidate for advanced preclinical trials.

\section{ASSOCIATED CONTENT}

\section{Supporting Information}

Inhibitor synthesis, characterization, and experimental procedures for FP, SPR, cytotoxicity, and Western Blot studies. This material is available free of charge via the Internet at http:// pubs.acs.org.

\section{AUTHOR INFORMATION}

\section{Corresponding Author}

*E-mail: patrick.gunning@utoronto.ca.

\section{Author Contributions}

${ }^{\perp}$ A.A.C., A.M.L., and M.G. contributed equally to this work.

\section{Funding}

Financial support was provided by a Canada Research Chair (PTG), an NSERC Discovery Grant (PTG), NIH R21CA186945 (PTG, HLG), the Princess Margaret Foundation (MDM), an Ontario Graduate Scholarship (AAC), an NSERC Graduate Scholarship (CEB), and an NSERC USRA (DBD).

Notes

The authors declare no competing financial interest.

\section{REFERENCES}

(1) Hasinoff, B. B.; Patel, D. The lack of target specificity of small molecule anticancer kinase inhibitors correlated with their ability to damage myocytes in vitro. Toxicol. Appl. Pharmacol. 2010, 249, 13239.

(2) Mouhayar, E.; Durand, J. B.; Cortes, J. Cardiovascular toxicity of tyrosine kinase inhibitors. Expert. Opin. Drug Saf. 2013, 12, 687-96.

(3) Yang, B.; Papoian, T. Tyrosine kinase inhibitor (TKI)-induced cardiotoxicity: approaches to narrow the gaps between preclinical safety evaluation and clinical outcome. J. Appl. Toxicol. 2012, 32, 94551.

(4) Engelman, J. A.; Settleman, J. Acquired resistance to tyrosine kinase inhibitors during cancer therapy. Curr. Opin. Genet. Dev. 2008, $18,73-79$.

(5) Barouch-Bentov, R. Mechanisms of drug-resistance in kinases. Expert Opin. Invest. Drug 2011, 2, 153-208.

(6) Daub, H.; Specht, K.; Ullrich, A. Strategies to overcome resistance to targeted protein kinase inhibitors. Nat. Rev. Drug. Discovery 2004, 3, 1001-10.

(7) Shyh-Han, T.; Nevaleinen, M. T. Signal transducer and activator of transcription $5 \mathrm{~A} / \mathrm{B}$ in prostate and breast cancers. Endocr. Relat. Cancer 2008, 15, 367-90.

(8) Becker, S.; Groner, B.; Muller, C. W. Three-dimensional structure of the Stat3beta homodimer bound to DNA. Nature 1998, 394, 14551.

(9) Chen, X.; Vinkemeier, U.; Zhao, Y.; Jeruzalmi, D.; Darnell, J. E.; Kuriyan, J. Crystal structure of a tyrosine phosphorylated Stat-1 dimer bound to DNA. Cell 1998, 93, 827-39.

(10) Nosaka, T.; Kawashima, T.; Misawa, K.; Ikuta, K.; Mui, A. L.; Kitamura, T. STAT5 as a molecular regulator of proliferation, differentiation and apoptosis in hematopoietic cells. EMBO J. 1999, $18,4754-65$.

(11) Cumaraswamy, A. A.; Todic, A.; Resetca, D.; Minden, M. D.; Gunning, P. T. Inhibitors of Stat5 protein signaling. MedChemComm 2012, 3, 22-27.

(12) Muller, J.; Sperl, B.; Reindl, W.; Kiessling, A.; Berg, T. Discovery of chromone-based inhibitors of the transcription factor Stat5. ChemBioChem 2008, 9, 723-27. 
(13) Nelson, E. A.; Walker, S. R.; Xiang, M.; Weisberg, E.; Bar-Natan, M.; Barrett, R.; Liu, S.; Kharbanda, S.; Christie, A. L.; Nicolais, M.; Griffin, J. D.; Stone, R. M.; Kung, A. L.; Frank, D. A. The STAT5 inhibitor pimozide displays efficacy in models of acute myelogenous leukemia driven by FLT3 mutations. Blood 2011, 117, 3421-29.

(14) Walker, S. R; Xiang, M.; Frank, D. A. Distinct roles of STAT3 and STAT5 in the pathogenesis and targeted therapy of breast cancer. Mol. Cell. Endocrinol. 2014, 382, 616-21.

(15) Muller, J.; Schust, J.; Berg, T. A high-throughput assay for signal transducer and activator of transcription $5 \mathrm{~b}$ based on fluorescence polarization. Anal. Biochem. 2008, 375, 249-54.

(16) Page, B. D.; Khoury, H.; Laister, R. C.; Fletcher, S.; Vellozo, M.; Manzoli, A.; Yue, P.; Turkson, J.; Minden, M. D.; Gunning, P. T. Small molecule STAT5-SH2 domain inhibitors exhibit potent antileukemia activity. J. Med. Chem. 2012, 55, 1047-55.

(17) Andricopulo, A. D.; Salum, L. B.; Abraham, D. J. Structure-based drug design strategies in medicinal chemistry. Curr. Top. Med. Chem. 2009, 9, 771-90.

(18) Kraskouskaya, D.; Duodu, E.; Arpin, C. C.; Gunning, P. T. Progress towards the development of $\mathrm{SH} 2$ domain inhibitors. Chem. Soc. Rev. 2013, 42, 3337-70.

(19) Liu, B. A.; Engelmann, B. W.; Nash, P. D. The language of SH2 domain interactions defines phosphotyrosine-mediated signal transduction. FEBS Lett. 2012, 586, 2597-2605.

(20) Morris, G. M.; Goodsell, D. S.; Halliday, R.; Huey, R.; Hart, W. E.; Belew, R. K.; Olson, A. J. Automated docking using a lamarckian genetic algorithm and empirical binding free energy function. $J$. Comput. Chem. 1998, 19, 1639-62.

(21) Ren, Z.; Mao, X.; Mertens, C.; Krishnaraj, R.; Qin, J.; Mandal, P. K.; Romanowski, M. J.; McMurray, J. S.; Chen, X. Crystal structure of unphosphorylated stat 3 core fragment. Biochem. Biophy. Res. Commun. 2008, 374, 1-5.

(22) Park, I.; Chenglong, L. Characterization of molecular recognition of STAT3 $\mathrm{SH} 2$ domain inhibitors through molecular simulation. J. Mol. Recognit. 2011, 24, 254-65. 